\title{
ANÁLISIS DE LAS RESTRICCIONES INTERNAS Y SU INCIDENCIA EN LA COMPETITIVIDAD DE LAS MYPES DEL SECTOR CONFECCIONES TEXTILES DEL DISTRITO DE TACNA, PERIODO 2007- 2008.
}

\author{
Autora: Elena Pomareda Angulo' \\ Universidad Privada de Tacna \\ E-mail: elenap40@yahoo.com.ar
}

\section{RESUMEN}

El trabajo corresponde a una investigación básica, de naturaleza descriptiva correlacional debido a que está basado en especificar propiedades, caracteristicas y determinar la relación de las variables de estudio: restricciones internas y competitividad de las MYPES del Sector Confecciones. Para tal propósito se consideró la información obtenida a través de la aplicación del cuestionario a 100 empresarios de las micro y pequeñas empresas del distrito de Tacna a fin de conocer las restricciones internas y la competitividad de las mismas.

El objetivo fue determinar de qué manera inciden las restricciones internas en la competitividad de las MYPES del sector confecciones textiles, del distrito de Tacna, en el periodo 2007- 2008.

Los resultados más importantes son las restricciones internas que inciden en la competitividad de las MYPES del sector confecciones textiles (las limitadas capacidades gerenciales, las escasas capacidades operativas y el bajo nivel de tecnologia) evidenciándose sus limitadas capacidades empresariales. Los directivos, en su mayoría, han sido formados empíricamente; no desarrollan habilidades gerenciales para la mejor solución de la problemática existente; tales como: dirigir un equipo de trabajo, enfrentar situaciones dificiles de disciplina y de intereses que pudieran surgir en el grupo, logro de resultados.

Las MYPES se caracterizan por su bajo nivel de competitividad, debido a su bajo nivel de productividad; puesto que los empresarios no han hecho eficiente uso de los recursos (insumos) al producir sus productos.

\section{PALABRAS CLAVE:}

MYPES del sector confecciones, restricciones internas, competitividad, productividad.

\begin{abstract}
This is a basic research descriptive and correlational research work, because it is based on specifying properties, characteristics and relationship between variables studied, namely internal restrictions and competitiveness of MYPES in clothing manufacturing area. We included information collected through a questionnaire applied to 100 microbusinessmen in the small and micro enterprises in Tacna district in order to determine the existing internal restrictions and their competitiveness.

Our objective was to determine how it is that internal restrictions influence on competitiveness of MYPES in clothing manufacturing areas in the district of Tacna in the study period 2007-2008.

The most important results are that internal restrictions that influence mainly on competitiveness in clothing manufacturing area are: limitation in managerial capacities, scarce operative capacities and low technology levels. This is evidenced on limited business capabilities. Most CEOs are empiric, they don't develop managerial capabilities to solve existing problems, such as leading a working team, deal with hard discipline situations and also deal with interests that appear in group and also achievement of results.

MYPES are characterized by their low level of competitiveness due to their low productivity since businessmen in charge have not made efficient use of their resources (supplies) when manufacturing their products.
\end{abstract}

\section{KEYWORDS:}

MYPES, clothing, internal restrictions, competitiveness, productivity. 


\section{INTRODUCCIÓN}

Las MYPES, del sector confecciones formalmente constituidas en la ciudad de Tacna son aproximadamente 135; de las cuales 100 han sido utilizadas como muestra de nuestro estudio, las que presentan limitadas capacidades empresariales; ya que sus directivos no desarrollan habilidades gerenciales; siendo su formación empírica.

Las MYPES, del Sector confecciones se caracterizan además por sus escasas capacidades operativas, puesto que desarrollan sus actividades con infraestructura en su modalidad de estructura adaptada; y cuenta en su mayoría, con equipamiento mínimo (máquina recta, remalladora y cortadora). Estas empresas se caracterizan por su bajo nivel de tecnología debido a que realizan sus operaciones de manera manual y no sistematizadamente. Asimismo, se encontró que la recepción y envío de pedidos son inadecuados; asi como también la conectividad vendedor comprador. Por lo tanto, estos resultados han incidido en su bajo nivel de competitividad.

Siendo la pregunta de investigación ¿De qué manera inciden las Restricciones Internas en la competitividad de las MYPES del Sector Confecciones Textiles del distrito de Tacna, en el período 2007-2008?; la que responde a la siguiente hipótesis: Las restricciones internas inciden directamente en la competitividad de las MYPES del sector confecciones del distrito de Tacna, en el período 2007-2008.

Conscientes de que las MYPES del sector confecciones textiles, es un sector que contribuye al desarrollo de la economía de nuestra región; y por ende, de nuestro país; la investigación permitirá dar alcances a los empresarios y a las instituciones encargadas para abordar y resolver la problemática existente.

\section{MATERIALESY MÉTODOS}

El presente estudio adoptó un diseño no experimental, de tipo descriptivo correlacional. Se considera no experimental; por cuanto no se manipula la variable independiente restricciones internas, es decir, se observa tal y como se da en su contexto natural para luego analizarla. Es de tipo descriptivo correlacional; porque se busca medir las variables (restricciones internas y competitividad) y la relación existente entre ambas. La población de estudio Comprende el Sector Confecciones del distrito de Tacna, conformado por 135 microempresas, cuya muestra constituye 100 microempresas; aplicándose para la recolección de datos el cuestionario, mediante la técnica de la encuesta. El instrumento de medición ha sido elaborado de acuerdo a las variables de estudio; el cual fue sometido a la prueba de confiabilidad y validez; para su análisis e interpretación se adoptó un diseño de análisis estadístico descriptivo e inferencial.

Los resultados alcanzados fueron los siguientes:

\section{RESULTADOS}

\section{RESTRICCIONES INTERNAS DE LAS MYPES DEL SECTOR CONFECCIONES TEXTILES DEL DISTRITO DE TACNA}

Gráfico $\mathrm{N}^{\circ} 01$

Capacidad Gerencial: Nivel de Conocimientos

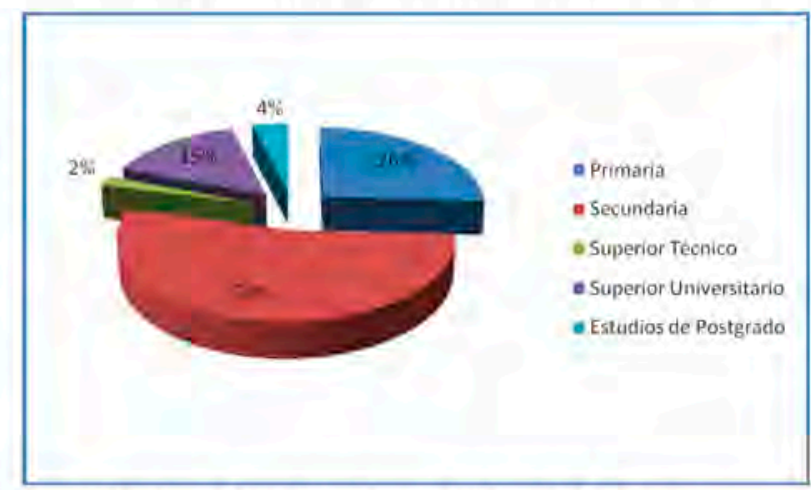

Fuente: Cuestionario Restricciones Internas. Elaboración: Propia

El $53 \%$ de los empresarios tiene estudios de nivel Secundario, el $26 \%$ de nivel de Primaria, el $15 \%$ de nivel de Superior Universitario y el $4 \%$ de nivel de Postgrado.

Esto se debe en gran medida a que los pequeños y microempresarios son empiricos, puesto que han adquirido conocimientos en el quehacer diario. $\mathrm{Y}$ en su mayoria al terminar su secundaria se han visto en la necesidad de realizar actividades que anteriormente han sido desarrolladas por sus familiares, de los cuales han heredado los conocimientos y habilidades necesarias para efectuar dichas labores. 
Gráfico $\mathrm{N}^{\circ} 02$

Habilidades: Trabaja en equipo, soluciona problemas y logra resultados

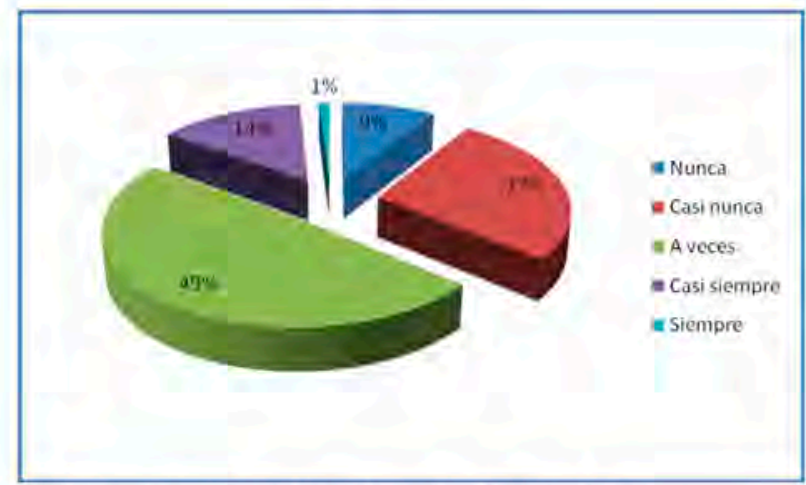

Fuente: Cuestionario Restricciones Internas. Elaboración: Propia

El $49 \%$ de los encuestados manifestó que "a veces" tiene la capacidad de dirigir un equipo de trabajo, solucionar problemas y lograr resultados; el $27 \%$ manifestó que casi nunca lo hacía; el $14 \%$ señaló que casi siempre está en capacidad para dirigir un equipo de trabajo, solucionar problemas y lograr resultados; el $9 \%$ indicó que nunca lo hacía; y, tan sólo el $1 \%$ manifestó que siempre lo hacía.

De por sí la palabra "equipo" implica la inclusión de más de una persona, lo que significa que el objetivo planteado no puede ser logrado $\sin$ la ayuda de todos sus miembros.

\section{Gráfico $\mathrm{N}^{\circ} 03$}

Actitudes y Valores: Ante la competitividad existente en el sector, ha establecido una visión y proyección para el desarrollo de sus actividades empresariales.

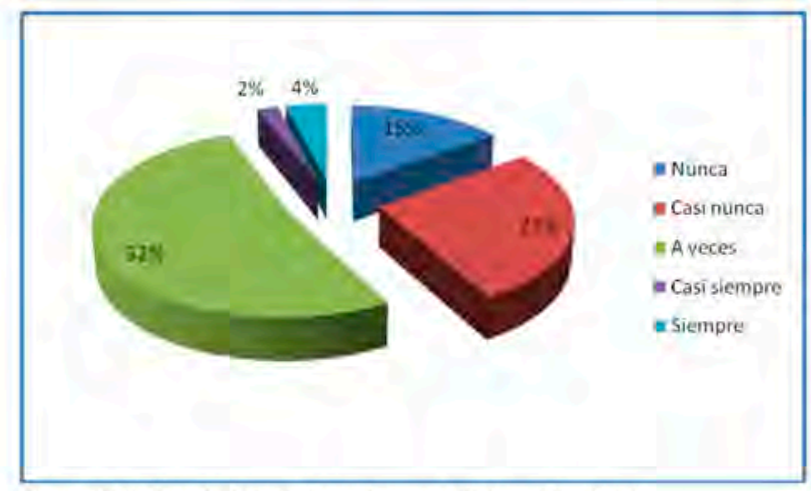

Fuente: Cuestionario Restricciones Internas. Elaboración: Propia

En el gráfico se observa que el $62 \%$ de los encuestados manifestó que ante la competitividad existente en el sector, "a veces" ha establecido una visión y proyección para el desarrollo de sus actividades empresariales.

\section{Capacidad Operativa: Infraestructura}

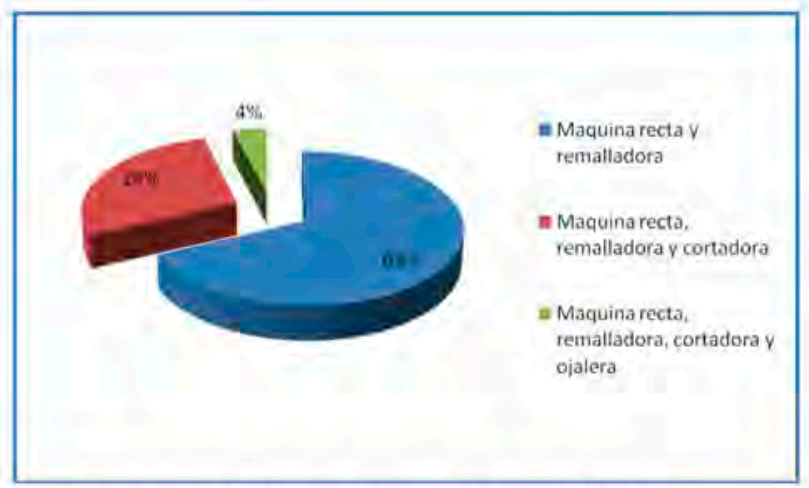

Fuente: Cuestionario Restricciones Internas. Elaboración: Propia

El 75\% de los encuestados, señaló que la infraestructura en la que desarrollan sus actividades corresponde al tipo de estructura adaptada; el 18\% manifestó que el tipo de su infraestructura era semiadaptada; y tan sólo el 7\% afirmó que el tipo de infraestructura con la que contaba era semiplanificada. Esto se debe a que la mayoría de Mypes tienen una infraestructura adaptada, realizando sus actividades en sus respectivos domicilios.

\section{Gráfico $\mathrm{N}^{\circ} 05$}

Capacidad Operativa: Equipamiento/Maquinaria.

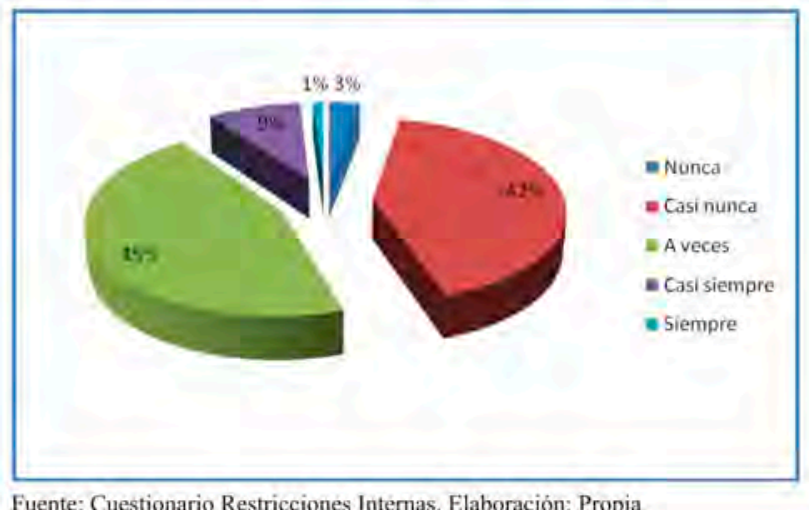

El $68 \%$ de los encuestados manifestó que el equipamiento y maquinaria que cuenta para el desarrollo de sus actividades consta de máquina recta y remalladora. La cual es utilizada para unir las piezas de la prenda a confeccionar. 


\section{Gráfico $\mathrm{N}^{\circ} 06$}

Capacidad Operativa: Realización de actividades de control de calidad producto terminado.

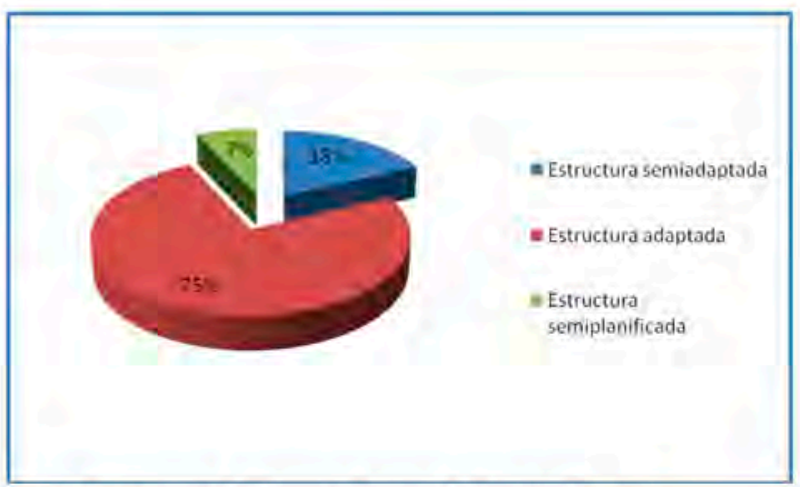

Fuente: Cuestionario Restricciones Internas, Elaboración: Propia

El 45\% de los encuestados, manifestaron que "a veces", considera realizar actividades de control de calidad producto terminado, el $42 \%$ manifestó que casi nunca lo hacía, el $9 \%$ de los encuestados manifestó que casi siempre lo hacía y un mínimo del $1 \%$ de los encuestados manifestó que siempre lo hacía. La calidad es lograr la satisfacción del cliente, cumpliendo con sus expectativas, necesidades, deseos o requerimientos.

\section{Gráfico $\mathrm{N}^{\circ} 07$}

Capacidad Tecnológica: Desarrollo de Actividades.

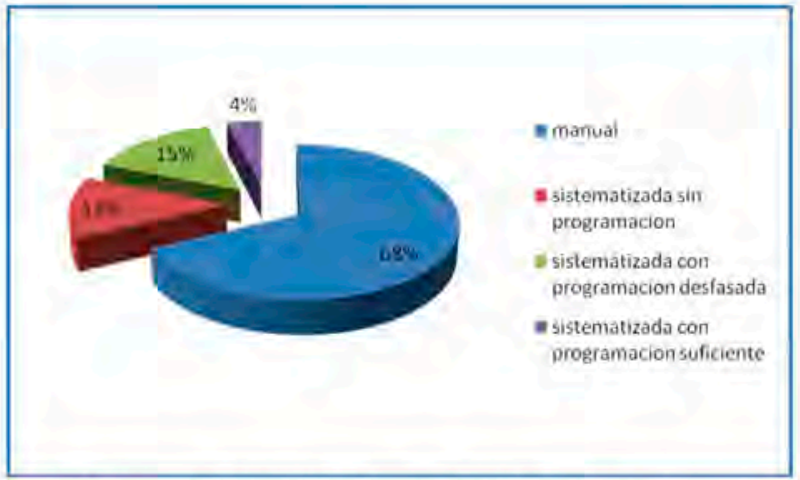

Fuente: Cuestionario Restricciones Internas. Elaboración: Propia

El $68 \%$ de los empresarios, manifestó que realizan sus actividades de manera manual. Las MYPES del sector confecciones textiles, en su mayoría ha optado por realizar sus actividades económicas de manera "manual"; no haciendo uso de las tecnologías de la información para el desarrollo de sus actividades económicas diarias.
Gráfico $\mathrm{N}^{\circ} 08$

Capacidad Tecnológica: Conectividad vendedor comprador:

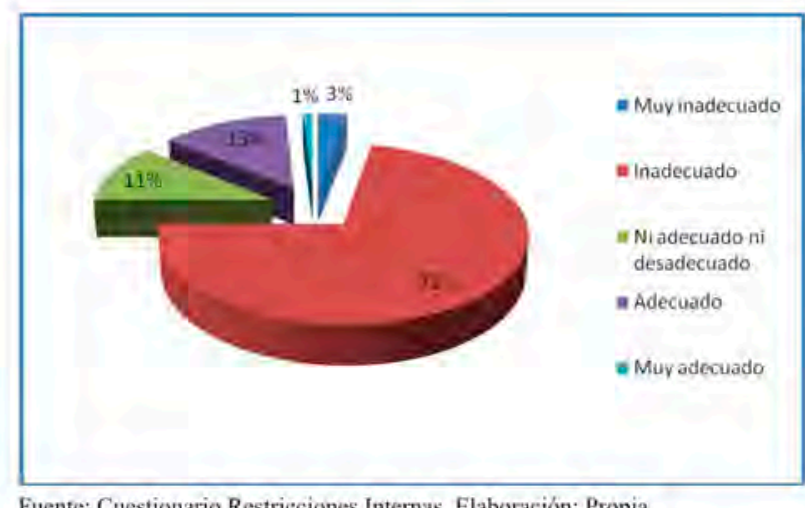

El $72 \%$ de los empresarios, manifestó que la conectividad vendedor comprador es inadecuada; al no emplearse los medios de comunicación entre el vendedor y el cliente; lo que no permite el adecuado desarrollo de sus actividades y operaciones.

\section{RESULTADOS}

\section{COMPETITIVIDAD DE LAS MYPES DEL SECTOR CONFECCIONES DEL DISTRITO DE TACNA}

\section{Gráfico $N^{\circ} 09$}

Innovación: Se realiza una mejora en los acabados de los productos existentes.

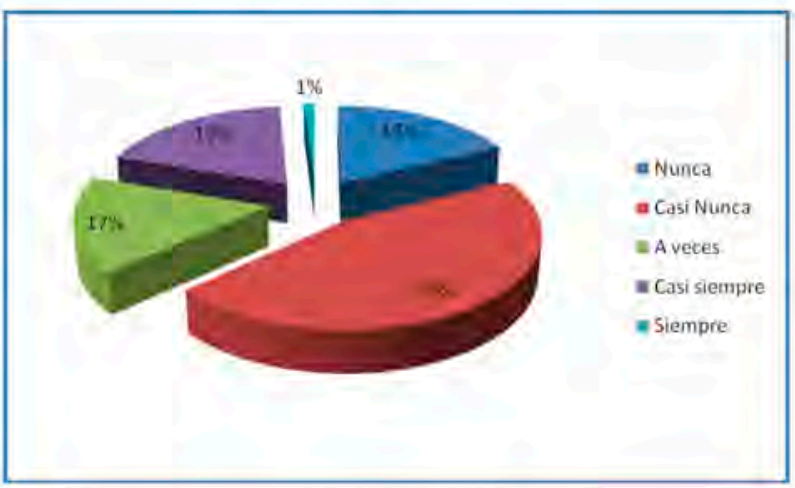

Fuente: Cuestionario Competitividad MYPES. Elaboración: Propia

El $46 \%$ de los empresarios encuestados manifestó que "casi nunca" se realiza una mejora en los acabados de los productos existentes.

Estos resultados se deben a los elevados costos que origina la mejora en los acabados y debido a la competitividad existente no se puede incurrir en ellos. 
Gráfico $\mathrm{N}^{\circ} 10$

Innovación: Se considera una evaluación desde la concepción de la idea hasta el lanzamiento del producto al mercado.

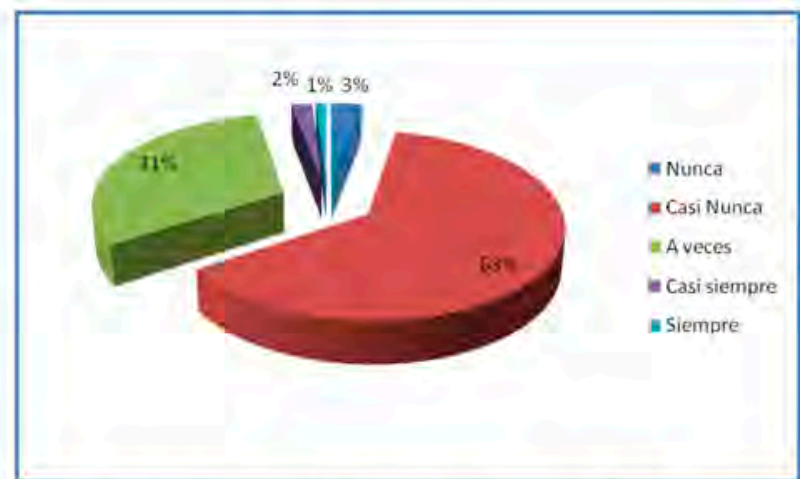

Fuente: Cuestionario Competitividad MYPES. Elaboración: Propia

El $64 \%$ de los encuestados manifestó que casi nunca se considera una evaluación desde la concepción de la idea hasta el lanzamiento del producto al mercado. Esta actividad incluye la definición detallada de las funciones y las especificaciones del producto, de las partes y los sistemas que la componen.

\section{Gráfico $\mathrm{N}^{\circ} 11$}

Innovación: Se considera el rediseño a los productos ofertados.

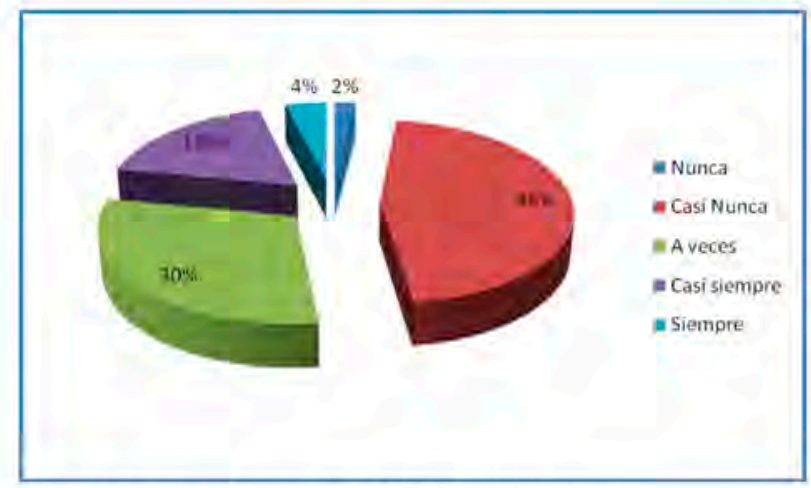

Fuente: Cuestionario Competitividad MYPES, Elaboración: Propia

El $46 \%$ de los encuestados indicó que "casi nunca" consideraba el rediseño a los productos ofertados. Con el fin alcanzar un nivel competitivo, las empresas del sector confecciones textiles, deben considerar como ventaja competitiva, la innovación a través del rediseño de los productos ofertados.

\section{Gráfico $\mathrm{N}^{\circ} 12$}

Innovación: Se considera nuevos procesos y mejoras a los productos, de acuerdo a las exigencias del sector.

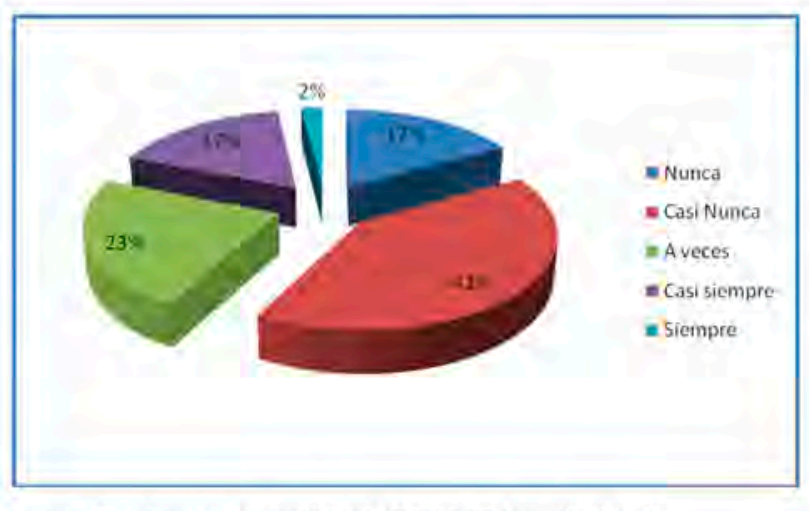

Fuente: Cuestionario Competitividad MYPES. Elaboración: Propia

El $41 \%$ de los encuestados señaló que casi nunca se consideraban nuevos procesos y mejoras a los productos, de acuerdo a las exigencias del sector. La redefinición de procesos obliga a replantearse todos los aspectos relacionados con un proceso, comenzando por los resultados del proceso hasta los propósitos iniciales.

\section{Gráfico $\mathrm{N}^{\circ} 13$}

Productividad: Tipo de Producto.

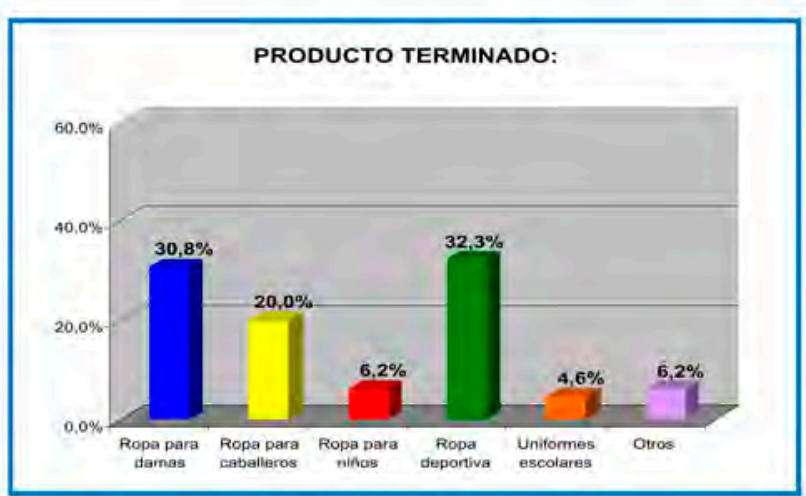

Fuente: PRODUCE TACNA, Elaboración: Propia

El 32,20\% de las Mypes se dedica a la confección de ropa deportiva, el $30,8 \%$ a la ropa de damas, el $20,00 \%$ a lá ropa de caballeros, el $4,60 \%$ a uniformes escolares y el $6,00 \%$ a ropa para niños; mientras que un significativo $6,00 \%$ pertenece al rubro de otros. 
Gráfico $\mathrm{N}^{\circ} 14$

Productividad: Monto de ventas

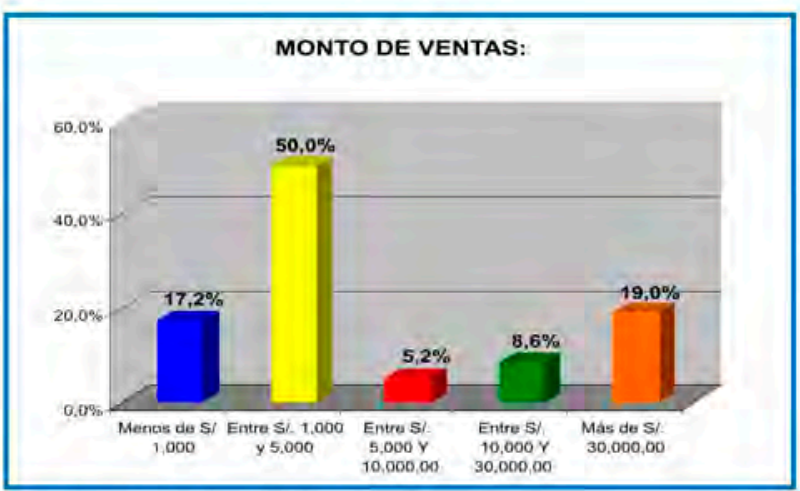

Fuente: PRODUCE TACNA. Elaboración: Propis

En cuanto al monto de ventas el ingreso mensual del $50,00 \%$ de las Mypes oscila entre S/. 1000.00 y S/. 5 000,00 ; el $5,20 \%$ entre S/. 5000.00 y S/. 10000.00 ; el $8,60 \%$ entre S/. 10000.00 y S/. 30000.00 y el $19,00 \%$ tiene ingresos mensuales por encima de los $\mathrm{S} / .30000 .00$.

\section{DISCUSIÓN}

Las Restricciones internas que inciden en la competitividad de las MYPES del Sector Confecciones Textiles son: Ias limitadas capacidades gerenciales, las escasas capacidades operativas y el bajo nivel de tecnología.

Los microempresarios dirigen de manera empírica; por lo que se observa la ausencia del trabajo en equipo, capacidad para solucionar problemas y lograr resultados, hacer frente a situaciones dificiles de disciplina y de intereses que pudieran surgir en el grupo, no propiciando nuevos enfoques y acciones.

Además se puede determinar que sus limitadas capacidades gerenciales se deben a que muestran ciertas actitudes y valores que no les permite desarrollarse competitivamente en el sector de las confecciones textiles.

Las MYPES del sector confecciones textiles se caracterizan por su bajo nivel de tecnología debido a que realizan sus operaciones de manera manual y no sistematizadamente. Las MYPES del sector confecciones se caracterizan por su bajo nivel de competitividad, debido a su bajo nivel de productividad; puesto que los empresarios no han hecho eficiente uso de los recursos (insumos) al producir sus productos.
Asimismo, se ha podido determinar que las MYPES del sector confecciones no consideran la innovación como fuente de ventaja competitiva, ya que no la consideran para el desarrollo de aspectos tales como: generación de nuevos procesos, desarrollo de producto, redefinición de los procesos productivos y comerciales.

Por lo tanto, se determinó que las restricciones internas incidieron directamente en la competitividad de las MYPES del Sector Confecciones del distrito de Tacna, en el periodo 2007 y 2008.

\section{REFERENCIAS}

1. Barreto C. Tarquino y García M. Martín (2005). Modelo Asociativo para el mejoramiento de la Competitividad de la Pequeña y Mediana Empresa del sector confección del Estado Lara, Venezuela. Revista Venezolana de Economía Social $N^{\circ} 10$.

2. Brito, Xiomara y otros (2001) Casos Simulados de Redes Empresariales entre Pequeñas y Medianas Empresas (PyMEs) en Barquisimeto (Fase Teórica). Trabajo de Grado presentado para optar al Título de Licenciado en Contaduria Pública. Decanato de Administración y Contaduria (DAC) Universidad Centroccidental Lisandro Alvarado (UCLA)

3. Instituto Nacional de Estadística e Informática. Perú Compendio Estadístico 2005, Lima 2005

4. Instituto Nacional de Estadistica e Informática. Compendio Cuentas Nacionales del Perú - Producto Bruto Interno por Departamentos 1994-2001. Lima, Febrero 2003.

5. Consejo Regional MYPE. Diagnóstico Regional Tacna de la Micro y Pequeña Empresa.

6. Gerry Jonson y Kevan Scholes. (2001).Dirección Estratégica. Edic. Pearson Educación, Madrid.

7. Jaime Garcia Díaz. Determinantes del Empleo Adecuado en las MYPE's en el Perú 1995-1998. Patrocinado por; BID, BM. Auspiciado por MECOVI - PERU

8. La Constitución Política del Perú de 1993.

9. Ley de Promoción y Formalización de la Micro y Pequeña Empresa "MYPE", Ley 28015. Lima Promulgada 3 julio 2003.

10. Ley de Promoción del Desarrollo Económico y Productivo, Ley 28304. Lima Promulgada 26 Julio 2004

11. Reglamento de la Ley 28015, mediante D.S. 0092003-TR. Lima Promulgada 11 Setiembre 2003.

12. Warren J. Keegan. Marketing Global. Edic. Prentice Hall, Madrid 1997. 